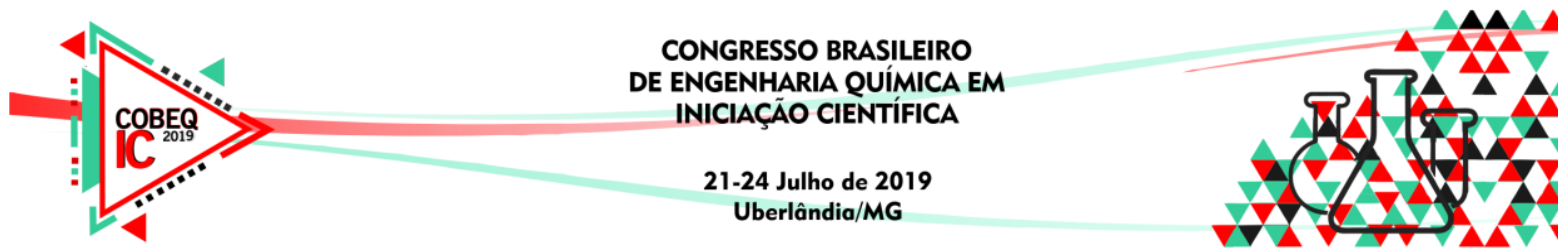

\title{
INFLUÊNCIA DO PH NA REMOÇÃO DO ANTIBIÓTICO OFLOXACINO POR ADSORÇÃO PELA ARGILA BENTONÍTICA VERDE-LODO CALCINADA
}

\author{
F. R. MARTINS ${ }^{1}$, R. ANTONELLI ${ }^{1}$, M. G. C.SILVA ${ }^{1}$ e M. G. A.VIEIRA ${ }^{1 *}$ \\ ${ }^{1}$ Universidade Estadual de Campinas, Faculdade de Engenharia Química \\ *E-mail para contato: melissagav@ feq.unicamp.br
}

\begin{abstract}
RESUMO - A presença de diferentes fármacos em águas de efluentes de esgoto tratado é uma preocupação crescente, dado os impactos ambientais que isto causa. A presença de antibióticos, sobretudo, preocupa devido à possibilidade do desenvolvimento de bactérias resistentes aos mesmos, além dos efeitos em animais e nos seres humanos. Sendo assim, o surgimento de métodos alternativos, para substituir os tradicionais, ganham cada vez mais destaque. Entre estes, a adsorção é um método eficaz que emergiu por apresentar vantagens como a fácil operação e o baixo custo e assim, deve-se estudar as melhores condições para aumentar sua eficácia. Nesse cenário, este projeto avaliou a influência do $\mathrm{pH}$ na adsorção do antibiótico ofloxacino pela argila Verde-lodo calcinada. Os testes apontaram que o pH inicial da solução não influenciou na remoção do antibiótico e apresentou resultados significativos de remoção $(>99,5 \%)$ para toda a faixa de $\mathrm{pH}$ estudada.
\end{abstract}

\section{INTRODUÇÃO}

A preocupação com a qualidade da água e conscientização com a presença de poluentes cresceu nos últimos anos (RAGASSI, 2018). A presença de contaminantes na água traz conseqüências aos animais aquáticos e silvestres, mas também pode afetar até mesmo os seres humanos (LEITE et. al., 2010). Além disso, preocupa-se com o desenvolvimento de bactérias resistentes à medicamentos (JOÃO, 2011). Dentre os contaminantes, já foram encontrados mais de 36 tipos de fármacos (JOÃO, 2011), sendo $15 \%$ pertencentes à classe dos antibióticos (SANTOS et. al., 2011). Os métodos tradicionais de tratamento de efluentes, tais como coagulação, floculação, decantação, filtração e desinfecção não são eficientes para remoção destes fármacos (LIMA et. al., 2017). Grande parte dos fármacos possuem grupos funcionais polares, como ácido carboxílico e amina, e, por isso, tendem a permanecer na fase aquosa, alcançando os ambientes aquáticos (MELO et. al., 2009). Dessa forma, um processo alternativo que pode ser usado para o tratamento dos efluentes contendo fármacos é a adsorção que se destaca por ser um processo simples e de fácil operação (HARO, 2017), além de não introduzir subprodutos ao ambiente (ROSSNER et. al., 2009).

O uso de argilas como adsorvente apresentou resultados na remoção de compostos orgânicos, nanocompósitos poliméricos, fluidos tixotrópicos (LOPER; PENHA, 2011), corantes (REHMAN et. al., 2013) e antibióticos (JALIL et. al., 2005). Além dos resultados satisfatórios como adsorvente, a argila é um composto natural abundante, com estabilidade 


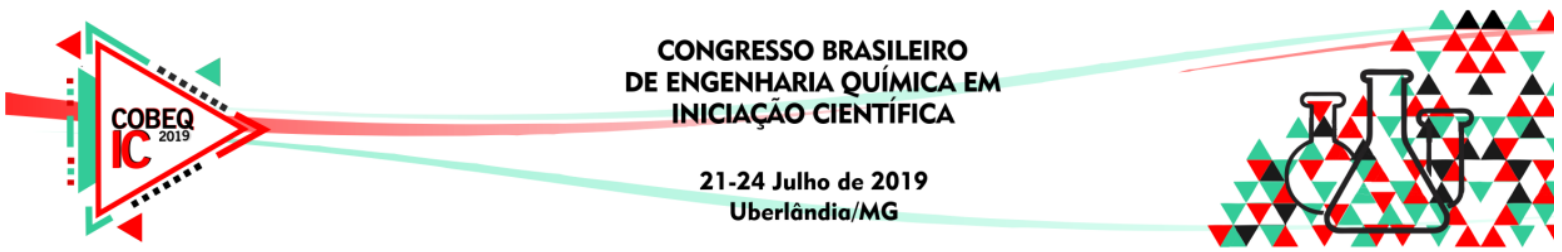

químico-mecânica e de baixo custo (PARK, 2013). As argilas podem ser modificadas de forma a aumentar sua capacidade de adsorção. De forma geral, as argilas bentoníticas são secas e moídas para se alcançar a granulometria adequada e, posteriormente, são calcinadas para serem usadas como adsorventes (BERTAGNOLLI, 2010).

O ofloxacino é um antibiótico sintético de amplo-espectro, pertencente à classe das fluoroquinolonas, usado principalmente no combate da tuberculose (GARCIA, 2013). O ofloxacino é excretado majoritariamente pela urina (ZANCHETTA et. al., 2015) e apresenta baixa biodegrabilidade, atingindo águas superficiais (PRADO, 2007). O antibiótico já foi detectado em concentrações acima das permitidas em águas superficiais em diferentes países, como Brasil,Austrália, França, Finlândia e Estados Unidos (SILVA, 2017). Dessa forma, a adsorção para o tratamento de efluentes contendo este fármaco visa diminuir os impactos ambientais.

Para se obter a maior eficiência do processo de adsorção, parâmetros como a afinidade entre adsorvente e adsorbato, temperatura, granulometria do adsorvente e $\mathrm{pH}$ devem ser levados em consideração (NASCIMENTO et. al., 2014). Dentre entre parâmetros, o pH da solução pode influenciar o rendimento da adsorção uma vez que, além de controlar a carga superficial do adsorvente, o pH também altera a ionização do adsorbato, de forma que modifica seu grau de dissociação e de protonação. Dessa forma, o pH modifica as interações eletrostáticas entre o par adsorvente - adsorbato, alterando a eficácia da adsorção (HARO, 2017).Diante do exposto, o objetivo deste trabalho consistiu em analisar a influência do $\mathrm{pH}$ da solução na adsorção do antibiótico ofloxacino utilizando a argila bentonítica Verde-lodo calcinada como adsorvente alternativo.

\section{METODOLOGIA}

\subsection{Contaminante farmacêutico}

A solução do contaminante emergente antimicrobiano ofloxacino (Tabela 1), fornecido pela empresa EMS Farmacêutica (CampinasıSP, Brasil), foi preparada utilizando água ultrapura de osmose reversa (modelo OS20LXE, Gehaka, Brasil).

Tabela 1 -Propriedades do ofloxacino

\begin{tabular}{|c|c|}
\hline \multicolumn{2}{|c|}{ Contaminante emergente } \\
\hline Fórmula molecular & $2 \mathrm{C}_{18} \mathrm{H}_{20} \mathrm{FN}_{3} \mathrm{O}_{4}$ \\
\hline Peso molecular $\left(\mathrm{g} \cdot \mathrm{mol}^{-1}\right)$ & 361,37 \\
\hline Número CAS & [82419-36-1] \\
\hline Constante de ionização (pKa) & 6,08 e 8,25 \\
\hline Solubilidade em água $\left(\mathrm{mg} \mathrm{L}^{-1}\right)$ & $28,3 \mathrm{mg} . \mathrm{L}^{-1}\left(25^{\circ} \mathrm{C}\right)$ \\
\hline
\end{tabular}

Fonte: Adaptado de ESPOSITO et al., 2017.

Os valores de pKa para o ofloxacino são 6,08 (pKa1) e 8,25 (pKa2), conforme Figura 1, e correspondem ao grupo ácido carboxílico e ao grupo amina na porção piperazina, respectivamente. Quando o $\mathrm{pH}$ é menor que 6,08; a molécula de ofloxacino pode ser encontrada como cátion $\left(\mathrm{ofx}^{+}\right)$devido à protonação de amino na parte da triazina. Entre $\mathrm{pH}$ de 6,08 e 8,25; o íon dipolar (ofx ${ }^{0}$ ) é a principal forma do ofloxacino devido à protonação de amino na parte da piperazina e à desprotonação docarboxila.Um ânion (ofx-) é uma espécie 


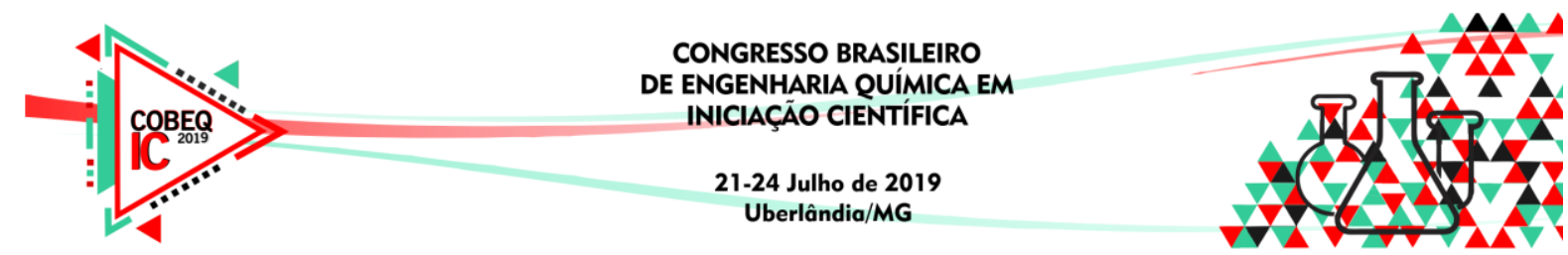

dominante quando o $\mathrm{pH}$ é maior que 8,25 , devido à perda do próton do grupo amino na porção piperazina.

Figura 1 -Distribuição de catiônica $\left(\right.$ ofx $\left.^{+}\right)$, neutra $\left(\right.$ofx $\left.^{0}\right)$ e aniônica $\left(\right.$ ofx $\left.^{-}\right)$do ofloxacino em solução aquosa em função do $\mathrm{pH}(\mathrm{pKa} 1=6,08$ e pKa2 $=8,25)$.

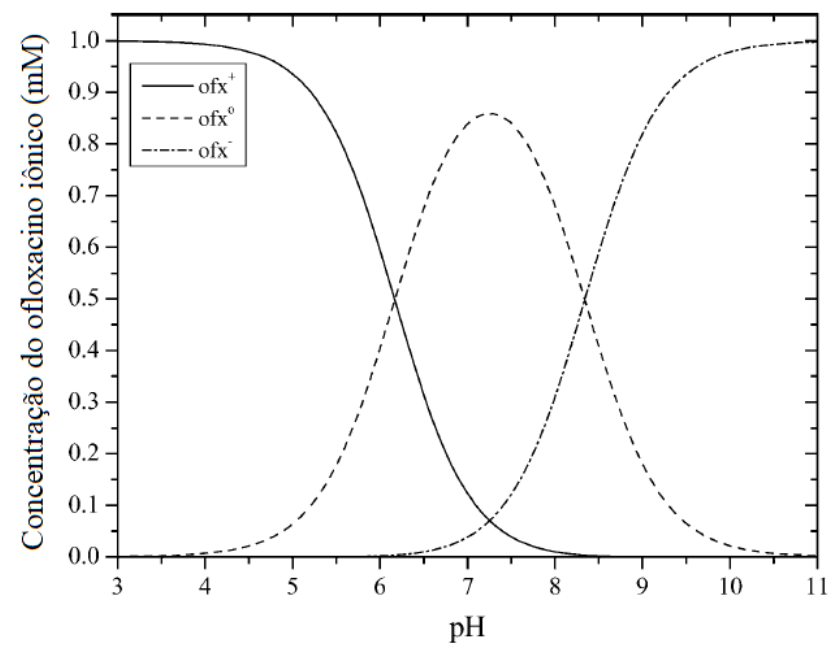

Fonte: GOYNE et. al., 2005.

\subsection{Adsorvente}

Como adsorvente foi usada a argila bentonítica Verde-lodo, fornecida na forma bruta pela empresa Dolomil Industrial LTDA (Boa Vista/PB).O adsorvente foi moído e classificado por peneiramento em peneiras Tyler (Granutest, Brasil) para obtenção de diâmetro médio de partícula de $0,855 \mathrm{~mm}$. Em seguida, a argila foi submetida à calcinação em mufla a $500{ }^{\circ} \mathrm{C}$ por um período de 24 horas, para obter maior estabilidade e para aumentar a capacidade de troca iônica.

\subsection{Ensaio da influência do pH}

Ensaios em triplicata foram conduzidos em erlenmeyers contendo $50 \mathrm{ml}$ da solução do fármaco antimicrobiano. Foi gotejada solução de ácido clorídrico ou de hidróxido de sódio a fim de se obter soluções com diferentes $\mathrm{pHs}(2,4,8,10$ e 11). Aos frascos foi adicionado 0,5 $\mathrm{g}$ do adsorvente e estes foram mantidos sob agitação constante de $200 \mathrm{rpm}$ em um Shaker por $24 \mathrm{~h}$ a temperatura constante de $25^{\circ} \mathrm{C}$. As amostras coletadas foram submetidas a centrifugação (modelo L600, Cence ${ }^{\circledR}$ ) a 4.000 rpm por período de 10 minutos e filtradas em filtros de seringa em teflon com membrana de politetrafluoroetileno hidrofilizada $(25 \mathrm{~mm} 0,45$ $\mu \mathrm{m})$ (Macherey-Nagel).A concentração final do antibiótico foi determinada por espectrofotometria UV-Visível (modelo Mini 1240, Shimadzu) no comprimento de onda de $350 \mathrm{~nm}$. A quantidade adsorvida $(q)$, em mmol.g ${ }^{-1}$, e a porcentagem de remoção (\%Rem) de remoção do fármaco ofloxacino foram calculadas pelas Equações 1 e 2:

$$
\begin{aligned}
& q=\frac{C_{0}-C_{f}}{m} \cdot V \\
& \% \text { Rem }=\left(\frac{C_{0}-C_{f}}{C_{0}}\right) .100
\end{aligned}
$$




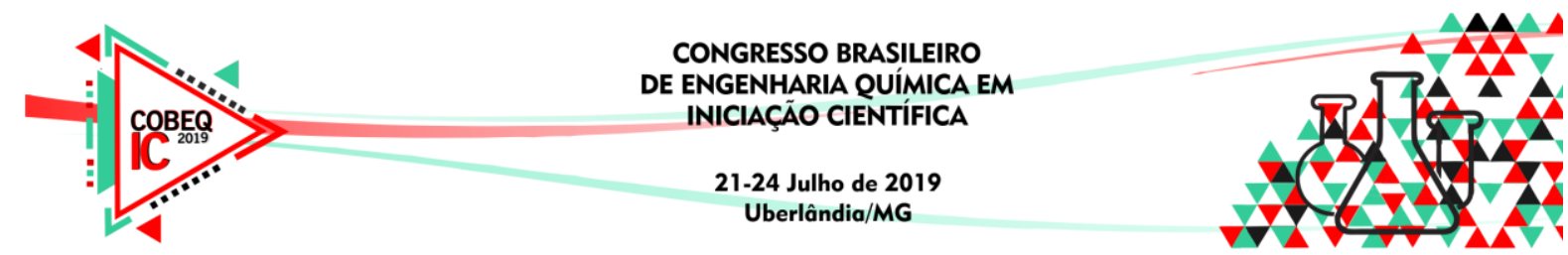

Onde:

$\mathrm{C}_{0}\left(\mathrm{mmol} \cdot \mathrm{L}^{-1}\right)$ : concentração inicial de fármaco na solução;

$\mathrm{C}_{\mathrm{f}}\left(\mathrm{mmol} \cdot \mathrm{L}^{-1}\right)$ : concentração após o processo de adsorção;

V (L): volume de solução de fármaco contida no Erlenmeyer;

m (g): massa de adsorvente.

\section{RESULTADOS E DISCUSSÃO}

A Tabela 2 apresenta as porcentagens de remoção e a quantidade adsorvida de cada ensaio para diferentes valores de $\mathrm{pH}$. Da tabela,pode-se observar que a remoção do antibiótico apresentou valores muito próximos para diferentes pHs.

Tabela 2-Porcentagem de remoção média para cada valor de $\mathrm{pH}$ analisado

\begin{tabular}{|c|c|c|}
\hline $\mathrm{pH}$ & Remoção (\%) & $\mathrm{q}\left(\mathrm{mg} \cdot \mathrm{g}^{-1}\right)$ \\
\hline \hline 2 & $99,68 \pm 0,06$ & $36,14 \pm 0,02$ \\
\hline 4 & $99,84 \pm 0,07$ & $36,14 \pm 0,02$ \\
\hline 8 & $99,59 \pm 0,10$ & $32,52 \pm 0,04$ \\
\hline 10 & $99,79 \pm 0,17$ & $36,14 \pm 0,06$ \\
\hline 11 & $99,84 \pm 0,13$ & $36,14 \pm 0,05$ \\
\hline
\end{tabular}

As porcentagens de remoção foram comparadas pelo Teste de Tukey, apresentando pvalor > 0,05 para todas as análises de forma que se pode afirmar que as médias da porcentagem de remoção para os diferentes pHs são estatisticamente iguais a $95 \%$ de confiança.Os dados da literatura mostram que o pH tem influencia na eficiência da adsorção em sistemas de ofloxacino com nanotubos de carbono (PENG et. al., 2012) e com alumina e sílica (GOYNE et. al., 2005). Na adsorção do ofloxacino com carbono ativado (KONGA et al., 2017) o pH inicial da solução aquosa teve um efeito importante, apresentando pH ótimo entre 5 e 7, com taxa de remoção de $91 \%$.O sistema utilizado nesse trabalho de adsorção, ofloxacino com a argila Verde-lodo, não apresenta dados disponíveis na literatura. Contudo, a partir do experimento e dos resultados obtidos para este par adsorvente - adsorbato, pode-se afirmar que o pH inicial da solução não influencia na porcentagem de remoção do antibiótico da solução, observando resultados significativos de remoção (> 99,5\%)para os diferentes pHs analisados. Desta forma, prefere-se trabalhar com $\mathrm{pH}$ neutro (na faixa de 6,5-7,5)com a finalidade de evitar gastos com reagentes para ajuste de $\mathrm{pH}$ da solução.

\section{CONCLUSÃO}

Os ensaios para avaliar a influência do $\mathrm{pH}$ inicial no processo de adsorção do antibiótico ofloxacino pela argila bentonítica Verde-lodo calcinada demonstraram resultados significativos de remoção maiores que $99,5 \%$ para todos os pHs avaliados.A partir dos resultados obtidos e aplicando o teste de Tukey, concluiu-se que para os diferentes pHs as médias de remoção do antibiótico são estatisticamente iguais. Assim, pode-se concluir que o pH inicial da solução não tem influência no processo de adsorção do antibiótico ofloxacino pela argila bentonítica Verde-lodo calcinada. Desta forma, não seria necessário controlar o pH 


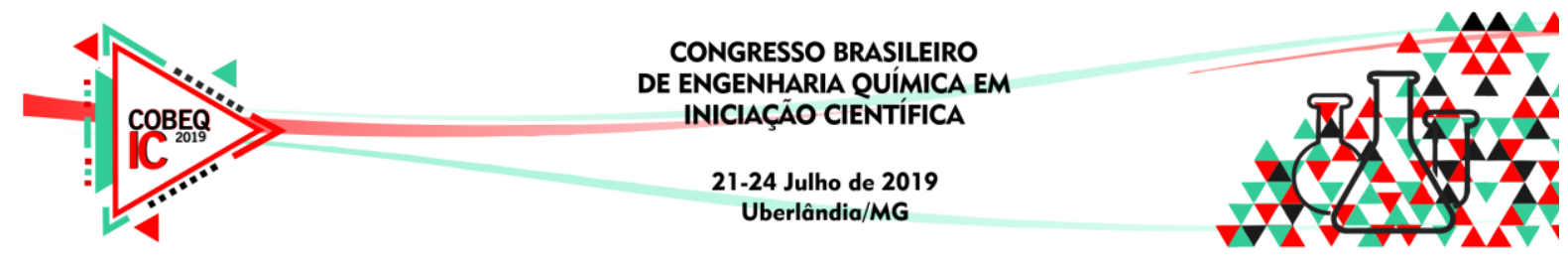

do meio para favorecer a adsorção, evitando gastos dispendiosos com reagentes ácidos e básicos, sendo possível efetuar a adsorção na faixa de $\mathrm{pH}$ em que o efluente contendo o contaminante emergente se encontra.

\section{AGRADECIMENTOS}

Agradecimentos à CAPES pela bolsa concedida à aluna de doutorado Raissa Antonelli, à FAPESP (Proc. 2016/05007-1) e ao CNPq (Proc. 406193/2018-5) pelo suporte financeiro, à empresa EMS Farmacêutica, pela doação do fármaco, e à empresa Dolomil Industrial LTDA, pela doação da argila.

\section{REFERÊNCIAS}

BERTAGNOLLI, C. Preparo e caracterização de argilas organofílicas para remoção de derivados do petróleo. 100 p. tese (Dissertação de mestrado). Unicamp, Campinas, 2010 .

ESPOSITO, B. R.; CAPOBIANCO, M. L.; MARTELLI, A.; NAVACCHIA, M. L.; PRETALI, L.; SARACINO, M.; ZANELLI, A.; EMMI, S. S. Advanced water remediation from ofloxacin by ionizing radiation. Radiat. Phys.Chem., v. 141, p. 118124, 2017.

GARCIA, M. C. A. Determinação da incerteza de medição na CLAE uma ferramenta da qualidade aplicada na análise de comprimidos de ofloxacino $400 \mathrm{mg} .110 \mathrm{p}$. tese (mestrado em Vigilância Sanitária). INCQS, Rio de Janeiro, 2013.

GOYNE, K. W., CHOROVER, J., KUBICKI, J. D., ZIMMERMAN, A. R., BRANTLEV, S.L. Sorption of the antibiotic ofloxacin to mesoporous and nonporous alumina and silica.J. Colloid Interf. Sci., v. 283, n. 1, p. 160-170, 2005.

HARO, N. K. Remoção dos fármacos Atenolol, Paracetamol e Ampicilina por adsorção em carvão ativado. $131 \mathrm{p}$. tese (Tese de doutorado). Universidade federal do Rio Grande do Sul, Porto Alegre, 2017.

JALIL, M. E. R.; BASCHINI, M.; SAPAG, K. Influence of pH and antibiotic solubility on the removal of ciprofloxacin from aqueous media using montmorillonite. Appl. Clay Sci., v. 114, p. 69-76, 2015.

JOÃO, W. S. J. Descarte de medicamentos. Pharm. Brasileira. Online, n. 82, p. 14-16, 2011.

KONGA, Q.; HE, X.; SHUC, L.; MIAO, M. Ofloxacin adsorption by activated carbon derived from luffa sponge: Kinetic, isotherm, and thermodynamic analyses. Process Saf. Environ., v. 112, p. 254-264, 2017.

LEITE, G. S.; AFONSO, R. J. C. F.; AQUINO, S. F. Caracterização de contaminantes presentes em sistemas de tratamento de esgotos, por cromatografia líquida acoplada à espectrometria de massas tandem em alta resolução. Quím. Nova, v. 33, n. 3, p. 734738, 2010. 


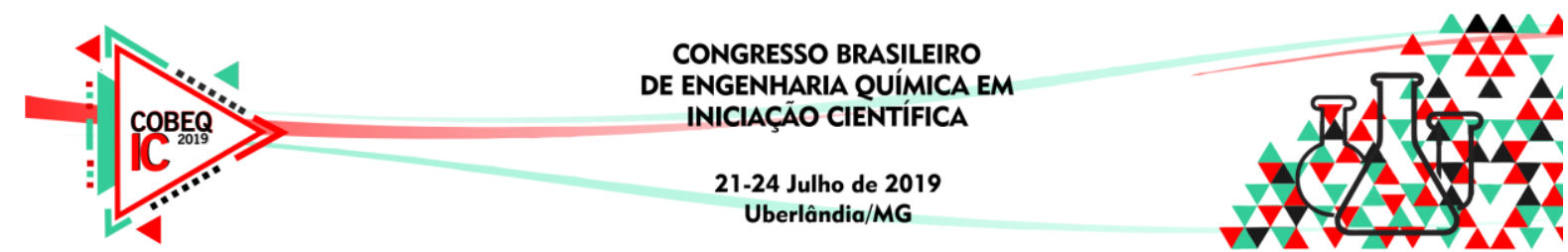

LIMA, D. R. S.; TONUCCI, M. C.; LIBÂNIO, M.; AQUINO, S. F. Fármacos e desreguladores endócrinos em águas brasileiras: ocorrência e técnicas de remoção. Eng. San.Amb. v. 22, n. 6, p. 1043-1054, nov. 2017.

MELO, S. A. S.; TROVÓ, A. G.; BAUTITZ, I. R.; NOGUEIRA, R. F. P. Degradação de fármacos residuais por processos oxidativos avançados. Quím. Nova. Sociedade Brasileira de Química, v. 32, n. 1, p. 188-197, 2009.

NASCIMENTO, R. F.; LIMA, A. C. A.; VIDAL, C. B.; RAULINO, G. S. C.Adsorção: aspectos teóricos e aplicações ambientais. 1 ed. Fortaleza: Imprensa Universitária, 2014.

PARK, Y.; AYOKO, G. A.; KURDI, R.; HORVARTH, E.; KRISTOF, J.; FROST, R. L. Adsorption of phenolic compounds by organoclays: Implications for the removal of organic pollutants from aqueous media. J. Colloid Interf. Sci., v. 406, p. 196-208, 2013.

PENG, H., PAN, B., LIU, M. W. R., ZHANG, D., WU, D., XING, B. Adsorption of ofloxacin on carbon nanotubes: Solubility, $\mathrm{pH}$ and cosolvent effects. J. Hazard. Mater., v. 211-212, p. 342-348, 2012.

PRADO, T. Avaliação da eficiência de um sistema de tratamento de efluente hospitalar por processo anaeróbio na remoção de coliformes, Pseudomonas aeruginosa, Klebsiella pneumoniae resistentes a antibióticos e Vírus da Hepatite A. 125 p. tese (Dissertação de mestrado). INCQS, Rio de Janeiro, 2007.

RAGASSI, B. Monitoramento de fármacos em água superficial e efluente de estação de tratamento de esgoto no município de dracena - SP. 54 p. Tese (Dissertação de mestrado). Unesp, Ilha Solteira, 2018.

REHMAN, M. S. U.; MUNIR, M.; ASHFAQ, M.; RASHID, N.; NAZAR, M. F.; DANISH, M.; HAN, J. I. Adsorption of Brilliant Green dye from aqueous solution on to red clay. Chem. Eng. J., v. 228, p. 54-62, 2013.

ROSSNER, A.; SNYDER, S. A.; KNAPPE, D. R. U. Removal of emerging contaminants of concern by alternative adsorbents. Water Research, v. 43, p. 3787-3796, 2009.

SANTOS, L.H.M.L.M.; ARAÚJO, A.N.; FACHINI, A.; PENA, A.; DELERUEMATOS, C.; MONTENEGRO, M.C.B.S.M. Ecotoxicological aspects related to the presence of pharmaceuticals in the aquatic environment. J. Hazard. Mater., v. 175, p. 45-95. 2010.

SILVA, R. P. Avaliação da remoção de antibióticos em águas naturais utilizando os processos de clarificação e adsorção. 90 p. tese (Dissertação de mestrado), UFOP,Ouro Preto, 2017.

ZANCHETTA, P. G.; PENA, A.; GONÇALVES, R. F. Desenvolvimento e validação de método para quantificação simultânea de ofloxacina, norfloxacina e ciprofloxacina em urina humana. Eng. San. e Amb., v. 20, n. 2, p.307-314, 2015. 